\title{
Evaluating the reduction of cancer pain using the infra- red negative ions amethyst Bio Mat in 12 subjects over 6 months
}

\author{
George Grant* \\ Richmond Hill, Ontario L4C 9H8 Canada
}

\begin{abstract}
The amethyst Bio Mat was used by 12 subjects for one hour three times per week as well as using the Detox Salt during sleep daily over 6 months period. We used two different biofeedback devices to measure pain reduction, Complete blood count (CBC) 12 hours fasting blood test including white blood cell count (WBC) and high-sensitivity C-reactive protein (HS-CRP) to measure inflammation. The Far Infrared/Negative Ions Amethyst Bio Mat reduced pain by 21\% and reduced HSCRP by $15 \%$ and reduced Stress by 52\% of 12 subjects in 6 months as validated by Pre and Post Biofeedback Brain Scans as well as fasting blood test to measure the stress hormone cortisol.
\end{abstract}

\section{Introduction}

Thermotherapy was enhanced when the Bio Mat was combined with the Detox salt during sleep to reduce pain and side effect of chemotherapy. The Bio Mat technology is a combination of far infrared rays, negative ion effects and the conductive properties of amethyst channels. These three powerful health stimulators are combined in a single, easy-to-use product with remarkable healing properties. The Bio Mat delivers soothing, deep-penetrating heat while stimulating the regeneration of damaged cells in the body. It's a safe and natural way to achieve optimal health now and maintain a stronger, more resilient body in the future. The combination of the bio-belt and the bio mat is a highly effective thermotherapy available to medical professionals and home consumers who want to reduce pain, stress and abdominal fat. The Bio Mat is an approved medical device by FDA [1-4].

The Bio Mat is a natural heating pad which lies on top of a massage table or a home mattress. It converts electricity through a computerized control panel, produced by Texas Instruments, into Far Infrared Rays (FIR), nature's invisible light. FIR was discovered by NASA to be the safest, most beneficial light wave. This reduces pain, swelling, increases blood flow and reduce stress by increasing the secretion of serotonin.

The Bio Mat also produces Negative Ions, nature's energizer, which deliver a molecular level massage. This accelerates and deepens all healing and cleansing processes. It balances $\mathrm{pH}$ by decreasing acidity and is considered the "Master Power Switch" which activates the body's entire cellular communication system, making every body function work better! Negative Ions alleviate allergies, migraines and sinus problems. These two components are transferred through Amethyst Quartz channels which cover the entire Bio Mat's surface. Amethyst Quartz is nature's Super Conductor, scientifically found to offer the steadiest, most powerful delivery of healthy far infrared light waves and the highest vibrational frequencies into the body.

The core of Bio Mat technology is a combination of far infrared rays [6-12 microns], negative ion effects and the conductive properties of amethyst channels. These three powerful health stimulators are combined in a single, easy-to-use product with remarkable healing properties. The Bio Mat manufactured and distributed by Richway International Inc. delivers soothing, deep-penetrating heat while stimulating the regeneration of damaged cells in the body. This highly effective therapy is now available to medical professionals and home consumers who want to improve health and well-being with products based on Nobel prize-winning scientific research pioneered by NASA and developed using pure, natural materials [5-7]. The Bio-Mat Professional is registered an FDA Medical Device \#2954299.

\section{Objectives of the study}

Examine the Benefits of the Bio Mat using the Infra-Red and Negative Ions for reducing pain in cancer patients for 12 subjects over 6 months. The main objective of the study is to measure the reduction of inflammation, joint pain and stiffness for 12 subjects suffering from cancer over 6 months using biofeedback devices and blood tests including HS-CRP that correlates with pain and inflammation.

\section{Methods}

12 subjects were tested before and after using the Bio Mat for one hour 3 times per week and sleep on the Bio Mat daily over 6 months. The biofeedback test for stress using ICAP Brain Scan, and the measurement for pain was done using the Bio Resonance Magnetic analyzer Biofeedback Device. The results were reduction in stress by $52 \%$ among subjects tested and an increased sense of well-being. The

Correspondence to: George F Grant, Ph.D., I.M.D, 17 Waterhouse Way, Richmond Hill, Ontario L4C 9H8 Canada, Tel: 416-562-3140; E-mail: drgrant@rogers.com

Received: February 26, 2016; Accepted: March 18, 2016; Published: March 21 , 2016 
pain was reduced by $21 \%$ and the HS-CRP was reduced by $15 \%$. All 12 subjects were tested at our clinic in Richmond Hill, ON Canada. The ICAP biofeedback device was used to measure stress reduction. The Bio Resonance Magnetic Analyzer Biofeedback Device was used to measure pain and inflammation. Blood test including HS-CRP was used to measure pain.

\section{Subject selection criteria}

12 subjects who were diagnosed with cancer and experienced moderate to severe pain were selected to participate in this case study and signed an informed consent. Subjects with medical, psychiatric conditions and those with heavy cancer medications were excluded from the study $[8,9]$.

Subjects were tested using Bio feedback Devices and blood tests before and after using the Bio Mat every week and a blood test to measure HS-CRP levels were obtained from each subject before and after 6 months at the completion of the case study.

\section{Biofeedback devices used to measure stress reduction \\ Quantum Resonance Magnetic Analyzer [QRMA]}

It measures electromagnetic waves emitted by human bodies which represent condition of cells, tissues and organs. The data is compared with standard spectrum to detect imbalances and measure stress reduction. This biofeedback device provides the stress of vital key organs and systems. Test Results provides a range of mild [0-30], moderate [30-60] and severe stress [70-100]. This correlates with DASS [Depression Anxiety Stress Scale] the International Stress Scale [10,11].

\section{ICAP [wireless Brain Scan EEG]}

ICAP Release Meter (Figure 1) is used to measure stress zone to monitor brain imbalance and blockages and Stress. The results also correlate with DASS. The ICAP ${ }^{\mathrm{m}}$ Release Meter System is made up of the EEG sensor, the signal transmitter, the USB base station that captures the signal, the proprietary algorithm that translates the raw data from the transmitter (Release Vector) and the visual representation of that data in the ICAP ${ }^{\mathrm{ra}}$ Release Meter software. The system also incorporates the Release ${ }^{\oplus}$ Technique, a method used to retrain the brain's responses. The device provides 3 distinct stress zones as well as an average stress score at the end of the measurement. A value of less than 500 indicates manageable stress, 500-700 medium stress and from 700 to 900 high stress. A value over 950 indicates extremely high stress [12,13].

\section{Blood test results HS-CEP}

Many scientists believe inflammation is a primary causative factor in many chronic diseases of today, such as diabetes, heart disease, cancer and degenerative brain disease. C-reactive protein (CRP) is

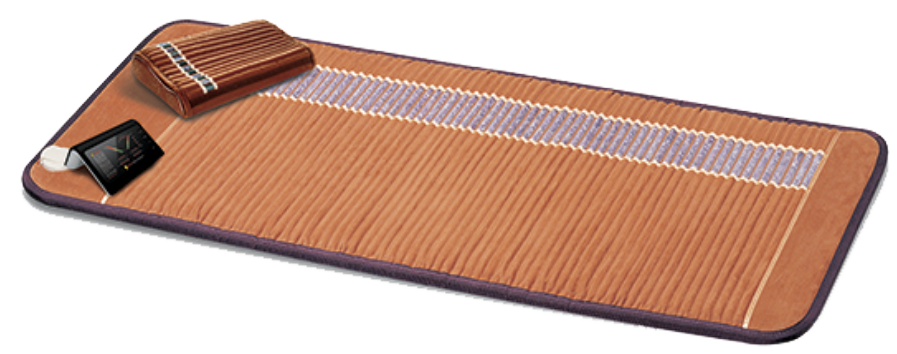

Figure 1. The Amethyst Negative Ions Bio Mat. produced by the liver. This protein was discovered in 1930 by William Tillett and Thomas Francis, investigators at the Rockefeller University. They found it could be isolated from the blood of patients with a specific type of pneumonia. Later it was discovered that elevated CRP-levels can be measured in blood in response to inflammation. The difference between CRP and HS-CRP is contained in the "HS" abbreviation; "high sensitivity". CRP is traditionally measured down to concentrations of 3-5 mg/L, whereas hs-CRP measures down to concentrations around $0.3 \mathrm{mg} / \mathrm{L}$. This improved sensitivity allows hs-CRP to be used to detect low levels of chronic inflammation.

\section{Results}

The summary of results is presented in Table 1.

\section{Discussion}

Subject \#1 [TC] male accountant mid sixty who is taking chemotherapy. He has improved after using the bio mat for 6 months with noticeable improvement in his pain and crp level.

Subject \#2[NB] male in his mid-seventy suffering from pain as a result of a cancer for 5 years. He did notice a difference in pain reduction in the first 8 weeks and after 12 weeks, he reported better sleep and less pain and less stress.

Subject\#3 [DL] a mid-forty female suffering from breast cancer. She had severe pain in her lymph which improved after 3-5 months test on the Bio mat. She also reported less pain and less stress as shown in her biofeedback scan, brain scan and her crp improved.

Subject\#4[TS] female mid-fifty with minor cancer pain and moderate sleep problem. She reported less stress and pain after only 7 weeks of using the bio mat as well as better sleep.

Table 1. Summary of Results.

\begin{tabular}{|l|c|c|}
\hline & Pain Scale 0 to 100 & Blood HS-CRP mg/L [3-5] \\
\hline Subject \#1[Pre] & 88 & 6.1 \\
\hline Subject \#1[post] & 68 & 3.5 \\
\hline Subject \#2[Pre] & 76 & 6.5 \\
\hline Subject \#2[post] & 57 & 4.2 \\
\hline Subject \#3[Pre] & 92 & 7.3 \\
\hline Subject \#3[post] & 73 & 5.6 \\
\hline Subject \#4[Pre] & 77 & 6.8 \\
\hline Subject \#4[post] & 53 & 4.8 \\
\hline Subject \#5[Pre] & 83 & 6.5 \\
\hline Subject \#5[post] & 37 & 4.9 \\
\hline Subject \#6[Pre] & 79 & 5.8 \\
\hline Subject \#6[post] & 66 & 3.8 \\
\hline Subject \#7[Pre] & 75 & 6.8 \\
\hline Subject \#7[post] & 54 & 5.7 \\
\hline Subject \#8[Pre] & 82 & 5.8 \\
\hline Subject \#8[post] & 67 & 4.8 \\
\hline Subject \#9[Pre] & 89 & 5.0 \\
\hline Subject \#9[post] & 67 & 4.1 \\
\hline Subject \#10[Pre] & 75 & 6.3 \\
\hline Subject \#10[post] & 65 & 4.1 \\
\hline Subject \#11[Pre] & 74 & 6.8 \\
\hline Subject \#11[post] & 52 & 3.3 \\
\hline Subject \#12[Pre] & & 6.8 \\
\hline Subject \#12[post] & 51 & \\
\hline
\end{tabular}


Subject\#5[VR] women in her early menopause that had cancer and pain. Her stress was high but felt less stress after 9 weeks of using the bio mat. She reportedless hot flash, increasedlibido, less pain and better sleep. Subject\#6 [IK] male in mid sixty with moderate pain but no medications. He has reported less pain, better sleep and less stress after using the bio mat but his pain remained mild during the 6 month study.

Subject\#7 [OL] female in mid seventy with high cancer pain but felt better after using the bio mat in 4 months. She was using pain killers but stopped taking medication after 9 weeks of using the bio mat. Subject\#8[RK] women in her early forty with no medication but moderate cancer pain and stress. Her stress and sleep habits improved dramatically in her first month of using the bio mat. Her CRP improved after 6 months of using the bio mat.

Subject\#9[ID] young male in his early forty with high pain and high stress after being diagnosed with prostate cancer. After using the bio mat for 6 months, his stress level was reduced and he noticed better sleep pattern with no need for sleep or pain medication. He stopped his chemotherapy after completing the study with moderate recovery.

Subject\#10[PL] female in her mid-sixty with lots of pain from cancer and stress. Her stress was high with poor sleeping habits. She experienced chest pain, back pain and she had made remarkable improvement after 6 months on the bio mat.

Subject \#11[CR] male in his early fifty with moderate cancer pain and very high stress and poor sleeping habits. His stress was improved after using the bio mat for 6 month.

Subject\#12 [SJ] female in her late sixty who takes 2 cancer medications, pain medications and sleep medication. After using the bio mat for 6 months she felt less pain, stress and reduced her medications by half. It appears from the above case study that the 12 subjects received above average improvement in pain reduction, stress reduction, better sleep, less discomfort and overall improvement particularly when they change also their lifestyle habits. The test results from the biofeedback devices correlated well with each other as well as with the blood test results.

Detecting inflammation can be tricky, but a marker called C-reactive protein (CRP), or [HS-CRP as measured] is released into the bloodstream by the liver when inflammation is present, and can be revealed in blood tests. Although doctors and scientists are still uncertain about the specifics around when the test should be done or who should have it, most clinicians use HS-CRP tests to monitor acute or chronic inflammation for cancer patients.

Pain is most often caused by the cancer itself. But pain can also be caused by cancer-related treatment or tests. You may also have pain that has nothing to do with the cancer or its treatment. Like anyone, you can get headaches, muscle strains, and other aches and pains.

\section{Pain from the cancer}

The amount of pain in Cancer Patients depends on the type of cancer, its stage (extent), and the pain threshold (tolerance for pain). People with advanced cancer are more likely to have pain. Pain from the cancer can be caused by a tumor pressing on bones, nerves, or body organs.

\section{Spinal cord compression}

When a tumor spreads to the spine, it can press on the spinal cord. This is called spinal cord compression. The first sign of compression is usually back and/or neck pain, sometimes with pain, numbness, or weakness in an arm or leg. Coughing, sneezing, or other movements often make it worse. If you have this pain, get help right away. This compression must be treated quickly to keep you from losing control of your bladder or bowel or being paralyzed. The cancer care team can treat the cause of the pain and give the patient medicine to help relieve the pain. The treatment for the compression soon after the pain begins, can usually avoid serious outcomes. Treatments usually involve radiation and steroids to shrink the tumor. Surgery can be done to remove a tumor that's pressing on the spine, which may then be followed by radiation.

Far Infrared bio mat/bio belt increases blood circulation and oxygen supply to damaged tissues (aiding reduction of chronic joint and muscle pain or sport injuries), promotes relaxation and comfort, induces sleep and relieves stress as shown in this case study.

Recently there have been reports detailing the hazards of exposure to certain kinds of electromagnetic fields, such as those from hightension power lines, cell phones, or from computer display terminals. Far Infrared heating systems have been tested in Japan and found free of toxic electromagnetic fields. The Swedish National Institute of Radiation Protection has also concluded that infrared heaters are not dangerous. Instead, Japanese researchers have reported that far infrared radiant heat antidotes the negative effects of toxic electromagnetic sources.

Further research is needed to elucidate the synergistic effect of using the bio mat/detox salt in several biological functions including the reduction of pain, stress, and reduction of CRP.

\section{Conflict of interest}

The author is not employed nor compensated by Richway International or Fuji Bio Sciences the manufacture and distributor of the bio -belt and bio mat. The company provided both the bio belt and the bio mat at no charge to conduct the case study for 12 subjects at our clinic in Toronto, ON Canada. The author has no financial interest in the company.

\section{References}

1. Alberts B (1990) The molecular biology of the cell ( $2^{\text {nd }}$ edtn) New York: Garland Press.

2. Kaltsas H (2009) What is ionization?

3. Grant George (2013) Your 101 ways to $101 \mathrm{Rx}$ : Healthy, wealthy, sexy and wise, 2013. The core Publishing Group.

4. Ricklan David (2006) 101 great ways to improve your life. Marlboro, NJ: SelfImprovement Online

5. Grant George (1996) The all in one guide to natural remedies.

6. Grant George (1995) Doctoral thesis, University of Toronto, Stress Factors affecting college educators.

7. Grant George (1992) Measuring stress affecting college professors. Ontario: Brock University.

8. Grant George (2015) Chicago International Pain Conference. Chicago, IL USA

9. Jones DP (2006) Redefining oxidative stress. Antioxid Redox Signal 8: 1865-1879. [Crossref]

10. Piller N (1999) Tribune Business News. The scientific basis and therapeutic benefits of far infrared ray therapy. Health Food Association 1-7.

11. Richardson W (1998) Spiritual values and gemstones. Marina Del Ray: Devorest Company. 
Grant G (2016) Evaluating the reduction of cancer pain using the infra-red negative ions amethyst Bio Mat in 12 subjects over 6 months

12. Lovibond PF, Lovibond SH (1995) The structure of negative emotional states: comparison of the Depression Anxiety Stress Scales (DASS) with the Beck Depression and Anxiety Inventories. Behav Res Ther 33: 335-343. [Crossref]
13. Bergonzini V, Salata C, Calistri A, Parolin C, Palù G (2010) View and review on viral oncology research. Infect Agent Cancer 5: 11. [Crossref]

Copyright: $(02016$ Grant G. This is an open-access article distributed under the terms of the Creative Commons Attribution License, which permits unrestricted use, distribution, and reproduction in any medium, provided the original author and source are credited. 